\title{
Quadruple Fixed Point Theorems under Nonlinear Contractive Conditions in Partially Ordered Metric Spaces
}

\author{
Erdal Karapinar, ${ }^{1}$ Wasfi Shatanawi, ${ }^{2}$ and Zead Mustafa ${ }^{2}$ \\ ${ }^{1}$ Department of Mathematics, Atılım University, 06836 Incek, Turkey \\ ${ }^{2}$ Department of Mathematics, The Hashemite University, Zarga 13115, Jordan
}

Correspondence should be addressed to Erdal Karapınar, erdalkarapinar@yahoo.com

Received 17 March 2012; Accepted 7 May 2012

Academic Editor: Debasish Roy

Copyright (C) 2012 Erdal Karapınar et al. This is an open access article distributed under the Creative Commons Attribution License, which permits unrestricted use, distribution, and reproduction in any medium, provided the original work is properly cited.

We prove a number of quadruple fixed point theorems under $\phi$-contractive conditions for a mapping $F: X^{4} \rightarrow X$ in ordered metric spaces. Also, we introduce an example to illustrate the effectiveness of our results.

\section{Introduction and Preliminaries}

The notion of coupled fixed point was initiated by Gnana Bhaskar and Lakshmikantham [1] in 2006. In this paper, they proved some fixed point theorems under a set of conditions and utilized their theorems to prove the existence of solutions to some ordinary differential equations. Recently, Berinde and Borcut [2] introduced the notion of tripled fixed point and extended the results of Gnana Bhaskar and Lakshmikantham [1] to the case of contractive operator $F: X \times X \times X \rightarrow X$, where $X$ is a complete ordered metric space. For some related works in coupled and tripled fixed point, we refer readers to [3-32].

For simplicity we will denote the cross product of $k \in \mathbb{N}$ copies of the space $X$ by $X^{k}$.

Definition 1.1 (see [2]). Let $X$ be a nonempty set and $F: X^{3} \rightarrow X$ a given mapping. An element $(x, y, z) \in X^{3}$ is called a tripled fixed point of $F$ if

$$
F(x, y, z)=x, \quad F(y, x, y)=y, \quad F(z, y, x)=z
$$


Let $(X, d)$ be a metric space. The mapping $\bar{d}: X^{3} \rightarrow X$, given by

$$
\bar{d}((x, y, z),(u, v, w))=d(x, y)+d(y, v)+d(z, w)
$$

defines a metric on $X^{3}$, which will be denoted for convenience by $d$.

Definition 1.2 (see [2]). Let $(X, \leq)$ be a partially ordered set and $F: X^{3} \rightarrow X$ a mapping. One says that $F$ has the mixed monotone property if $F(x, y, z)$ is monotone nondecreasing in $x$ and $z$ and is monotone nonincreasing in $y$; that is, for any $x, y, z \in X$,

$$
\begin{aligned}
& x_{1}, x_{2} \in X, \quad x_{1} \leq x_{2}, \text { implies } F\left(x_{1}, y, z\right) \leq F\left(x_{2}, y, z\right), \\
& y_{1}, y_{2} \in X, \quad y_{1} \leq y_{2}, \text { implies } F\left(x, y_{2}, z\right) \leq F\left(x, y_{1}, z\right), \\
& z_{1}, z_{2} \in X, \quad z_{1} \leq z_{2}, \text { implies } F\left(x, y, z_{1}\right) \leq F\left(x, y, z_{2}\right) .
\end{aligned}
$$

Let us recall the main results of [2] to understand our motivation toward our results in this paper.

Theorem 1.3 (see [2]). Let $(X, \leq)$ be a partially ordered set and $(X, d)$ a complete metric space. Let $F: X^{3} \rightarrow X$ be a continuous mapping such that $F$ has the mixed monotone property. Assume that there exist $j, k, l \in[0,1)$ with $j+k+l<1$ such that

$$
d(F(x, y, z), F(u, v, w)) \leq j d(x, u)+k d(y, v)+l d(z, w)
$$

for all $x, y, z, u, v, w \in X$ with $x \geq u, y \leq v$, and $z \geq w$. If there exist $x_{0}, y_{0}, z_{0} \in X$ such that $x_{0} \leq F\left(x_{0}, y_{0}, z_{0}\right), y_{0} \geq F\left(y_{0}, x_{0}, y_{0}\right)$, and $z_{0} \leq F\left(z_{0}, y_{0}, x_{0}\right)$, then $F$ has a tripled fixed point.

Theorem 1.4 (see [2]). Let $(X, \leq)$ be a partially ordered set and $(X, d)$ a complete metric space. Let $F: X^{3} \rightarrow X$ be a mapping having the mixed monotone property. Assume that there exist $j, k, l \in$ $[0,1)$ with $j+k+l<1$ such that

$$
d(F(x, y, z), F(u, v, w)) \leq j d(x, u)+k d(y, v)+l d(z, w)
$$

for all $x, y, z, u, v, w \in X$ with $x \geq u, y \leq v$, and $z \geq w$. Assume that $X$ has the following properties:

(i) if a nondecreasing sequence $x_{n} \rightarrow x$, then $x_{n} \leq x$ for all $n \in \mathbf{N}$,

(ii) if a nonincreasing sequence $y_{n} \rightarrow y$, then $y_{n} \geq y$ for all $n \in \mathbf{N}$.

If there exist $x_{0}, y_{0}, z_{0} \in X$ such that $x_{0} \leq F\left(x_{0}, y_{0}, z_{0}\right), y_{0} \geq F\left(y_{0}, x_{0}, y_{0}\right)$, and $z_{0} \leq F\left(z_{0}, y_{0}, x_{0}\right)$, then $F$ has a tripled fixed point.

Very recently, Karapinar introduced the notion of quadruple fixed point and obtained some fixed point theorems on the topic [33]. Extending this work, quadruple fixed point is developed and related fixed point theorems are proved in [34-39]. 
Definition 1.5 (see[34]). Let $X$ be a nonempty set and $F: X^{4} \rightarrow X$ a given mapping. An element $(x, y, z, w) \in X \times X^{3}$ is called a quadruple fixed point of $F$ if

$$
F(x, y, z, w)=x, \quad F(y, z, w, x)=y, \quad F(z, w, x, y)=z, \quad F(w, x, y, z)=w .
$$

Let $(X, d)$ be a metric space. The mapping $\bar{d}: X^{4} \rightarrow X$, given by

$$
\bar{d}((x, y, z, w),(u, v, h, l))=d(x, y)+d(y, v)+d(z, h)+d(w, l),
$$

defines a metric on $X^{4}$, which will be denoted for convenience by $d$.

Remark 1.6. In $[33,34,38]$, the notion of quadruple fixed point is called quartet fixed point.

Definition 1.7 (see[34]). Let $(X, \leq)$ be a partially ordered set and $F: X^{4} \rightarrow X$ a mapping. One says that $F$ has the mixed monotone property if $F(x, y, z, w)$ is monotone nondecreasing in $x$ and $z$ and is monotone nonincreasing in $y$ and $w$; that is, for any $x, y, z, w \in X$,

$$
\begin{aligned}
x_{1}, x_{2} \in X, & x_{1} \leq x_{2}, \text { implies } F\left(x_{1}, y, z, w\right) \leq F\left(x_{2}, y, z, w\right) \\
y_{1}, y_{2} \in X, & y_{1} \leq y_{2}, \text { implies } F\left(x, y_{2}, z, w\right) \leq F\left(x, y_{1}, z, w\right) \\
z_{1}, z_{2} \in X, & z_{1} \leq z_{2}, \text { implies } F\left(x, y, z_{1}, w\right) \leq F\left(x, y, z_{2}, w\right) \\
w_{1}, w_{2} \in X, & w_{1} \leq w_{2}, \text { implies } F\left(x, y, z, w_{2}\right) \leq F\left(x, y, z, w_{1}\right) .
\end{aligned}
$$

By following Matkowski [40], we let $\Phi$ be the set of all nondecreasing functions $\phi$ : $[0,+\infty) \rightarrow[0,+\infty)$ such that $\lim _{n \rightarrow+\infty} \phi^{n}(t)=0$ for all $t>0$. Then, it is an easy matter to show that

(1) $\phi(t)<t$ for all $t>0$,

(2) $\phi(0)=0$.

In this paper, we prove some quadruple fixed point theorems for a mapping $F: X^{4} \rightarrow$ $X$ satisfying a contractive condition based on some $\phi \in \Phi$.

\section{Main Results}

Our first result is the following.

Theorem 2.1. Let $(X, \leq)$ be a partially ordered set and $(X, d)$ a complete metric space. Let $F: X^{4} \rightarrow$ $X$ be a continuous mapping such that $F$ has the mixed monotone property. Assume that there exists $\phi \in \Phi$ such that

$$
d(F(x, y, z, w), F(u, v, h, l)) \leq \phi(\max \{d(x, u), d(y, v), d(z, h), d(w, l)\})
$$

for all $x, y, z, w, u, v, h, l \in X$ with $x \geq u, y \leq v, z \geq h$, and $w \leq l$. If there exist $x_{0}, y_{0}, z_{0}, w_{0} \in X$ such that $x_{0} \leq F\left(x_{0}, y_{0}, z_{0}, w_{0}\right), y_{0} \geq F\left(y_{0}, z_{0}, w_{0}, x_{0}\right), z_{0} \leq F\left(z_{0}, w_{0}, x_{0}, y_{0}\right)$ and $w_{0} \geq F\left(w_{0}, x_{0}, y_{0}, z_{0}\right)$, then $F$ has a quadruple fixed point. 
Proof. Suppose $x_{0}, y_{0}, z_{0}, w_{0} \in X$ are such that $x_{0} \leq F\left(x_{0}, y_{0}, z_{0}, w_{0}\right), y_{0} \geq F\left(y_{0}, z_{0}, w_{0}, x_{0}\right)$, $z_{0} \leq F\left(z_{0}, w_{0}, x_{0}, y_{0}\right)$, and $w_{0} \geq F\left(w_{0}, x_{0}, y_{0}, z_{0}\right)$. Define

$$
\begin{array}{ll}
x_{1}=F\left(x_{0}, y_{0}, z_{0}, w_{0}\right), & y_{1}=F\left(y_{0}, z_{0}, w_{0}, x_{0}\right), \\
z_{1}=F\left(z_{0}, w_{0}, x_{0}, y_{0}\right), & w_{1}=F\left(w_{0}, x_{0}, y_{0}, z_{0}\right) .
\end{array}
$$

Then, $x_{0} \leq x_{1}, y_{0} \geq y_{1}, z_{0} \leq z_{1}$, and $w_{0} \geq w_{1}$. Again, define $x_{2}=F\left(x_{1}, y_{1}, z_{1}, w_{1}\right)$, $y_{2}=F\left(y_{1}, z_{1}, w_{1}, x_{1}\right), z_{2}=F\left(z_{1}, w_{1}, x_{1}, y_{1}\right)$, and $w_{2}=F\left(w_{1}, x_{1}, y_{1}, z_{1}\right)$. Since $F$ has the mixed monotone property, we have $x_{0} \leq x_{1} \leq x_{2}, y_{2} \leq y_{1} \leq y_{0}, z_{0} \leq z_{1} \leq z_{2}$, and $w_{2} \leq w_{1} \leq w_{0}$. Continuing this process, we can construct four sequences $\left(x_{n}\right),\left(y_{n}\right),\left(z_{n}\right)$, and $\left(w_{n}\right)$ in $X$ such that

$$
\begin{aligned}
& x_{n}=F\left(x_{n-1}, y_{n-1}, z_{n-1}, w_{n-1}\right) \leq x_{n+1}=F\left(x_{n}, y_{n}, z_{n}, w_{n}\right) \\
& y_{n+1}=F\left(y_{n}, z_{n}, w_{n}, x_{n}\right) \leq y_{n}=F\left(y_{n-1}, z_{n-1}, w_{n-1}, x_{n-1}\right) \\
& z_{n}=F\left(z_{n-1}, w_{n-1}, x_{n-1}, y_{n-1}\right) \leq z_{n+1}=F\left(z_{n}, w_{n}, x_{n}, y_{n}\right) \\
& w_{n+1}=F\left(w_{n}, x_{n}, y_{n}, z_{n}\right) \leq w_{n}=F\left(w_{n-1}, x_{n-1}, y_{n-1}, z_{n-1}\right) .
\end{aligned}
$$

If, for some integer $n$, we have $\left(x_{n+1}, y_{n+1}, z_{n+1}, w_{n+1}\right)=\left(x_{n}, y_{n}, z_{n}, w_{n}\right)$, then $F\left(x_{n}, y_{n}, z_{n}, w_{n}\right)=x_{n}, F\left(y_{n}, z_{n}, w_{n}, x_{n}\right)=y_{n}, F\left(z_{n}, w_{n}, x_{n}, y_{n}\right)=z_{n}$, and $F\left(w_{n}, x_{n}, y_{n}, z_{n}\right)=$ $w_{n}$; that is, $\left(x_{n}, y_{n}, z_{n}, w_{n}\right)$ is a quadruple fixed point of $F$. Thus, we will assume that $\left(x_{n+1}, y_{n+1}, z_{n+1}, w_{n+1}\right) \neq\left(x_{n}, y_{n}, z_{n}, w_{n}\right)$ for all $n \in \mathbb{N}$; that is, we assume that $x_{n+1} \neq x_{n}, y_{n+1} \neq y_{n}$, or $z_{n+1} \neq z_{n}$ or $w_{n+1} \neq w_{n}$. For any $n \in \mathbb{N}$, we have

$$
\begin{aligned}
d\left(x_{n+1}, x_{n}\right) & :=d\left(F\left(x_{n}, y_{n}, z_{n}, w_{n}\right), F\left(x_{n-1}, y_{n-1}, z_{n-1}, w_{n-1}\right)\right) \\
& \leq \phi\left(\max \left\{d\left(x_{n}, x_{n-1}\right), d\left(y_{n}, y_{n-1}\right), d\left(z_{n}, z_{n-1}\right), d\left(w_{n}, w_{n-1}\right)\right\}\right) \\
d\left(y_{n}, y_{n+1}\right) & :=d\left(F\left(y_{n-1}, z_{n-1}, w_{n-1}, x_{n-1}\right), F\left(y_{n}, z_{n}, w_{n}, x_{n}\right)\right) \\
& \leq \phi\left(\max \left\{d\left(y_{n-1}, y_{n}\right), d\left(z_{n}, z_{n-1}\right), d\left(w_{n}, w_{n-1}\right), d\left(x_{n-1}, x_{n}\right)\right\}\right) \\
d\left(z_{n+1}, z_{n}\right) & :=d\left(F\left(z_{n}, w_{n}, x_{n}, y_{n}\right), F\left(z_{n-1}, w_{n-1}, x_{n-1}, y_{n-1}\right)\right) \\
& \leq \phi\left(\max \left\{d\left(z_{n}, z_{n-1}\right), d\left(w_{n}, w_{n-1}\right), d\left(x_{n}, x_{n-1}\right), d\left(y_{n}, y_{n-1}\right)\right\}\right) \\
d\left(w_{n}, w_{n+1}\right) & :=d\left(F\left(w_{n-1}, x_{n-1}, y_{n-1}, z_{n-1}\right), F\left(w_{n}, x_{n}, y_{n}, z_{n}\right)\right) \\
& \leq \phi\left(\max \left\{d\left(y_{n-1}, y_{n}\right), d\left(z_{n}, z_{n-1}\right), d\left(w_{n}, w_{n-1}\right), d\left(x_{n-1}, x_{n}\right)\right\}\right)
\end{aligned}
$$

From (2.4), it follows that

$$
\begin{aligned}
\max & \left\{d\left(x_{n+1}, x_{n}\right), d\left(y_{n}, y_{n+1}\right), d\left(z_{n+1}, z_{n}\right), d\left(w_{n}, w_{n+1}\right)\right\} \\
& \leq \phi\left(\max \left\{d\left(x_{n}, x_{n-1}\right), d\left(y_{n}, y_{n-1}\right), d\left(z_{n}, z_{n-1}\right), d\left(w_{n}, w_{n-1}\right)\right\}\right) .
\end{aligned}
$$


By repeating (2.5) $n$ times, we get that

$$
\begin{aligned}
\max & \left\{d\left(x_{n+1}, x_{n}\right), d\left(y_{n}, y_{n+1}\right), d\left(z_{n+1}, z_{n}\right), d\left(w_{n}, w_{n+1}\right)\right\} \\
& \leq \phi\left(\max \left\{d\left(x_{n}, x_{n-1}\right), d\left(y_{n}, y_{n-1}\right), d\left(z_{n}, z_{n-1}\right), d\left(w_{n}, w_{n-1}\right)\right\}\right) \\
& \leq \phi^{2}\left(\max \left\{d\left(x_{n-1}, x_{n-2}\right), d\left(y_{n-1}, y_{n-2}\right), d\left(z_{n-1}, z_{n-2}\right), d\left(w_{n-1}, w_{n-1}\right)\right\}\right) \\
& \vdots \\
& \leq \phi^{n}\left(\max \left\{d\left(x_{1}, x_{0}\right), d\left(y_{1}, y_{0}\right), d\left(z_{1}, z_{0}\right), d\left(w_{1}, w_{0}\right)\right\}\right) .
\end{aligned}
$$

Now, we will show that $\left(x_{n}\right),\left(y_{n}\right),\left(z_{n}\right)$, and $\left(w_{n}\right)$ are Cauchy sequences in $X$. Let $\epsilon>0$. Since

$$
\lim _{n \rightarrow+\infty} \phi^{n}\left(\max \left\{d\left(x_{1}, x_{0}\right), d\left(y_{1}, y_{0}\right), d\left(z_{1}, z_{0}\right), d\left(w_{1}, w_{0}\right)\right\}\right)=0
$$

and $\epsilon>\phi(\epsilon)$, there exist $n_{0} \in \mathbb{N}$ such that

$$
\phi^{n}\left(\max \left\{d\left(x_{1}, x_{0}\right), d\left(y_{1}, y_{0}\right), d\left(z_{1}, z_{0}\right), d\left(w_{1}, w_{0}\right)\right\}\right)<\epsilon-\phi(\epsilon) \quad \forall n \geq n_{0}
$$

This implies that

$$
\max \left\{d\left(x_{n+1}, x_{n}\right), d\left(y_{n}, y_{n+1}\right), d\left(z_{n+1}, z_{n}\right), d\left(w_{n}, w_{n+1}\right)\right\}<\epsilon-\phi(\epsilon) \quad \forall n \geq n_{0} .
$$

For $m, n \in \mathbb{N}$, we will prove by induction on $m$ that

$$
\max \left\{d\left(x_{n}, x_{m}\right), d\left(y_{n}, y_{m}\right), d\left(z_{n}, z_{m}\right), d\left(w_{n}, w_{m}\right)\right\}<\epsilon \quad \forall m \geq n \geq n_{0} .
$$

Since $\epsilon-\phi(\epsilon)<\epsilon$, then by using (2.9) we conclude that (2.10) holds when $m=n+1$. Now suppose that (2.10) holds for $m=k$. For $m=k+1$, we have

$$
\begin{aligned}
d\left(x_{n}, x_{k+1}\right) & \leq d\left(x_{n}, x_{n+1}\right)+d\left(x_{n+1}, x_{k+1}\right) \\
& \leq \epsilon-\phi(\epsilon)+d\left(F\left(x_{n}, y_{n}, z_{n}, w_{n}\right), F\left(x_{k}, y_{k}, z_{k}, w_{k}\right)\right) \\
& \leq \epsilon-\phi(\epsilon)+\phi\left(\max \left\{d\left(x_{n}, x_{k}\right), d\left(y_{n}, y_{k}\right), d\left(z_{n}, z_{k}\right), d\left(w_{n}, w_{k}\right)\right\}\right) \\
& <\epsilon-\phi(\epsilon)+\phi(\epsilon)=\epsilon .
\end{aligned}
$$

Similarly, we show that

$$
\begin{gathered}
d\left(y_{n}, y_{k+1}\right)<\epsilon, \\
d\left(z_{n}, z_{k+1}\right)<\epsilon, \\
d\left(w_{n}, w_{k+1}\right)<\epsilon .
\end{gathered}
$$


Hence, we have

$$
\max \left\{d\left(x_{n}, x_{k+1}\right), d\left(y_{n}, y_{k+1}\right), d\left(z_{n}, z_{k+1}\right), d\left(w_{n}, w_{k+1}\right)\right\}<\epsilon
$$

Thus, (2.10) holds for all $m \geq n \geq n_{0}$. Hence, $\left(x_{n}\right),\left(y_{n}\right),\left(z_{n}\right)$, and $\left(w_{n}\right)$ are Cauchy sequences in $X$.

Since $X$ is a complete metric space, there exist $x, y, z, w \in X$ such that $\left(x_{n}\right),\left(y_{n}\right)$, $\left(z_{n}\right)$ and $\left(w_{n}\right)$ converge to $x, y, z$, and $w$, respectively. Finally, we show that $(x, y, z, w)$ is a quadruple fixed point of $F$. Since $F$ is continuous and $\left(x_{n}, y_{n}, z_{n}, w_{n}\right) \rightarrow(x, y, z, w)$, we have $x_{n+1}=F\left(x_{n}, y_{n}, z_{n}, w_{n}\right) \rightarrow F(x, y, z, w)$. By the uniqueness of limit, we get that $x=$ $F(x, y, z, w)$. Similarly, we show that $y=F(y, z, w, x), z=F(z, w, x, y)$, and $w=F(w, x, y, z)$. So, $(x, y, z, w)$ is a quadruple fixed point of $F$.

By taking $\phi(t)=k t$, where $k \in[0,1)$, in Theorem 2.1, we have the following.

Corollary 2.2. Let $(X, \leq)$ be a partially ordered set and $(X, d)$ a complete metric space. Let $F: X^{4} \rightarrow$ $X$ be a continuous mapping such that $F$ has the mixed monotone property. Assume that there exists $k \in[0,1)$ such that

$$
d(F(x, y, z, w), F(u, v, h, l)) \leq k \max \{d(x, u), d(y, v), d(z, h), d(w, l)\}
$$

for all $x, y, z, w, u, v, h, l \in X$ with $x \geq u, y \leq v, z \geq h$, and $w \leq l$. If there exist $x_{0}, y_{0}, z_{0}, w_{0} \in X$ such that $x_{0} \leq F\left(x_{0}, y_{0}, z_{0}, w_{0}\right), y_{0} \geq F\left(y_{0}, z_{0}, w_{0}, x_{0}\right), z_{0} \leq F\left(z_{0}, w_{0}, x_{0}, y_{0}\right)$, and $w_{0} \geq$ $F\left(w_{0}, x_{0}, y_{0}, z_{0}\right)$, then $F$ has a quadruple fixed point.

As a consequence of Corollary 2.2, we have the following.

Corollary 2.3. Let $(X, \leq)$ be a partially ordered set and $(X, d)$ a complete metric space. Let $F: X^{4} \rightarrow$ $X$ be a continuous mapping such that $F$ has the mixed monotone property. Assume that there exist $a_{1}, a_{2}, a_{3}, a_{4} \in[0,1)$ with $a_{1}+a_{2}+a_{3}+a_{4}<1$ such that

$$
d(F(x, y, z, w), F(u, v, h, l)) \leq a_{1} d(x, u)+a_{2} d(y, v)+a_{3} d(z, h)+a_{4} d(w, l)
$$

for all $x, y, z, w, u, v, h, l \in X$ with $x \geq u, y \leq v, z \geq h$, and $w \leq l$. If there exist $x_{0}, y_{0}, z_{0}, w_{0} \in X$ such that $x_{0} \leq F\left(x_{0}, y_{0}, z_{0}, w_{0}\right), y_{0} \geq F\left(y_{0}, z_{0}, w_{0}, x_{0}\right), z_{0} \leq F\left(z_{0}, w_{0}, x_{0}, y_{0}\right)$ and $w_{0} \geq F\left(w_{0}, x_{0}, y_{0}, z_{0}\right)$, then $F$ has a quadruple fixed point. dropped.

By adding an additional hypothesis, the continuity of $F$ in Theorem 2.1 can be

Theorem 2.4. Let $(X, \leq)$ be a partially ordered set and $(X, d)$ a complete metric space. Let $F: X^{4} \rightarrow$ $X$ be a mapping having the mixed monotone property. Assume that there exists $\phi \in \Phi$ such that

$$
d(F(x, y, z, w), F(u, v, h, l)) \leq \phi(\max \{d(x, u), d(y, v), d(z, h), d(w, l)\})
$$

for all $x, y, z, w, u, v, h, l \in X$ with $x \geq u, y \leq v, z \geq h$, and $w \leq l$. Assume also that $X$ has 
the following properties:

(i) if a nondecreasing sequence $x_{n} \rightarrow x$, then $x_{n} \leq x$ for all $n \in \mathbf{N}$,

(ii) if a nonincreasing sequence $y_{n} \rightarrow y_{\text {, then }} y_{n} \geq y$ for all $n \in \mathbf{N}$.

If there exist $x_{0}, y_{0}, z_{0}, w_{0} \in X$ such that $x_{0} \leq F\left(x_{0}, y_{0}, z_{0}, w_{0}\right), y_{0} \geq F\left(y_{0}, z_{0}, w_{0}, x_{0}\right)$, $z_{0} \leq F\left(z_{0}, w_{0}, x_{0}, y_{0}\right)$, and $w_{0} \geq F\left(w_{0}, x_{0}, y_{0}, z_{0}\right)$, then $F$ has a quadruple fixed point.

Proof. By following the same process in Theorem 2.1, we construct four Cauchy sequences $\left(x_{n}\right),\left(y_{n}\right),\left(z_{n}\right)$, and $\left(w_{n}\right)$ in $X$ with

$$
\begin{gathered}
x_{1} \leq x_{2} \leq \cdots \leq x_{n} \leq \cdots, \\
y_{1} \geq y_{2} \geq \cdots \geq y_{n} \geq \cdots, \\
\mathrm{z}_{1} \leq z_{2} \leq \cdots \leq z_{n} \leq \cdots, \\
w_{1} \geq w_{2} \geq \cdots \geq w_{n} \geq \cdots,
\end{gathered}
$$

such that $x_{n} \rightarrow x \in X, y_{n} \rightarrow y \in X, z_{n} \rightarrow z \in X$, and $w_{n} \rightarrow w \in X$. By the hypotheses on $X$, we have $x_{n} \leq x, y_{n} \geq y, z_{n} \leq z$, and $w_{n} \geq w$ for all $n \in \mathbf{N}$. From (2.16), we have

$$
\begin{aligned}
d\left(F(x, y, z, w), x_{n+1}\right) & :=d\left(F(x, y, z, w), F\left(x_{n}, y_{n}, z_{n}, w_{n}\right)\right) \\
& \leq \phi\left(\max \left\{d\left(x, x_{n}\right), d\left(y, y_{n}\right), d\left(z, z_{n}\right), d\left(w, w_{n}\right)\right\}\right) \\
d\left(y_{n+1}, F(y, z, w, x)\right) & :=d\left(F\left(y_{n}, z_{n}, w_{n}, x_{n}\right), F(y, z, w, x)\right) \\
\leq & \phi\left(\max \left\{d\left(y_{n}, y\right), d\left(z_{n}, z\right), d\left(w_{n}, w\right), d\left(x_{n}, x\right)\right\}\right) \\
d\left(F(z, w, x, y), z_{n+1}\right) & :=d\left(F(z, w, x, y), F\left(z_{n}, w_{n}, x_{n}, y_{n}\right)\right) \\
\leq & \phi\left(\max \left\{d\left(x, x_{n}\right), d\left(y, y_{n}\right), d\left(z, z_{n}\right), d\left(w, w_{n}\right)\right\}\right) \\
d\left(w_{n+1}, F(w, x, y, z)\right) & :=d\left(F\left(w_{n}, x_{n}, y_{n}, z_{n}\right), F(w, x, y, z)\right) \\
& \leq \phi\left(\max \left\{d\left(y_{n}, y\right), d\left(z_{n}, z\right), d\left(w_{n}, w\right), d\left(x_{n}, x\right)\right\}\right)
\end{aligned}
$$

From (2.18), we have

$$
\max \left\{\begin{array}{l}
d\left(F(x, y, z, w), x_{n+1}\right), \\
d\left(y_{n+1}, F(y, z, w, x)\right), \\
d\left(F(z, w, x, y), z_{n+1}\right), \\
d\left(w_{n+1}, F(w, x, y, z)\right)
\end{array}\right\} \leq \phi\left(\max \left\{\begin{array}{l}
d\left(x, x_{n}\right), d\left(y, y_{n}\right), \\
d\left(z, z_{n}\right), d\left(w, w_{n}\right)
\end{array}\right\}\right) .
$$

Letting $n \rightarrow+\infty$ in (2.19), it follows that $x=F(x, y, z, w), y=F(y, z, w, x), z=F(z, w, x, y)$, and $w=F(w, x, y, z)$. Hence, $(x, y, z, w)$ is a quadruple fixed point of $F$.

By taking $\phi(t)=k t$, where $k \in[0,1)$, in Theorem 2.4, we have the following result. 
Corollary 2.5. Let $(X, \leq)$ be a partially ordered set and $(X, d)$ a complete metric space. Let $F: X^{4} \rightarrow$ $X$ be a mapping having the mixed monotone property. Assume that there exists $k \in[0,1)$ such that

$$
d(F(x, y, z, w), F(u, v, h, l)) \leq k \max \{d(x, u), d(y, v), d(z, h), d(w, l)\}
$$

for all $x, y, z, w, u, v, h, l \in X$ with $x \geq u, y \leq v, z \geq h$, and $w \leq l$. Assume also that $X$ has the following properties:

(i) if a nondecreasing sequence $x_{n} \rightarrow x$, then $x_{n} \leq x$ for all $n \in \mathbf{N}$,

(ii) if a nonincreasing sequence $y_{n} \rightarrow y_{\text {, then }} y_{n} \geq y$ for all $n \in \mathbf{N}$.

If there exist $x_{0}, y_{0}, z_{0}, w_{0} \in X$ such that $x_{0} \leq F\left(x_{0}, y_{0}, z_{0}, w_{0}\right), y_{0} \geq F\left(y_{0}, z_{0}, w_{0}, x_{0}\right), z_{0} \leq$ $F\left(z_{0}, w_{0}, x_{0}, y_{0}\right)$, and $w_{0} \geq F\left(w_{0}, x_{0}, y_{0}, z_{0}\right)$, then $F$ has a quadruple fixed point.

As a consequence of Corollary 2.5, we have the following.

Corollary 2.6. Let $(X, \leq)$ be a partially ordered set and $(X, d)$ a complete metric space. Let $F: X^{4} \rightarrow$ $X$ be a mapping having the mixed monotone property. Assume that there exist $a_{1}, a_{2}, a_{3}, a_{4} \in[0,1)$ with $a_{1}+a_{2}+a_{3}+a_{4}<1$ such that

$$
d(F(x, y, z, w), F(u, v, h, l)) \leq a_{1} d(x, u)+a_{2} d(y, v)+a_{3} d(z, h)+a_{4} d(w, l)
$$

for all $x, y, z, w, u, v, h, l \in X$ with $x \geq u, y \leq v, z \geq h$, and $w \leq l$. Assume that $X$ has the following properties:

(i) if a nondecreasing sequence $x_{n} \rightarrow x$, then $x_{n} \leq x$ for all $n \in \mathbf{N}$,

(ii) if a nonincreasing sequence $y_{n} \rightarrow y_{\text {, then }} y_{n} \geq y$ for all $n \in \mathbf{N}$.

If there exist $x_{0}, y_{0}, z_{0}, w_{0} \in X$ such that $x_{0} \leq F\left(x_{0}, y_{0}, z_{0}, w_{0}\right), y_{0} \geq F\left(y_{0}, z_{0}, w_{0}, x_{0}\right), z_{0} \leq$ $F\left(z_{0}, w_{0}, x_{0}, y_{0}\right)$, and $w_{0} \geq F\left(w_{0}, x_{0}, y_{0}, z_{0}\right)$, then $F$ has a quadruple fixed point.

Now we prove the following result.

Theorem 2.7. In addition to the hypotheses of Theorem 2.1 (resp., Theorem 2.4), suppose that

$$
\left[\left(x_{0} \leq y_{0}\right) \wedge\left(z_{0} \leq y_{0}\right) \wedge\left(x_{0} \leq w_{0}\right) \wedge\left(z_{0} \leq w_{0}\right)\right] \vee\left[\left(y_{0} \leq x_{0}\right) \wedge\left(y_{0} \leq z_{0}\right) \wedge\left(w_{0} \leq x_{0}\right) \wedge\left(w_{0} \leq z_{0}\right)\right]
$$

Then, $x=y=z=w$. 
Proof. Without loss of generality, we may assume that $x_{0} \leq y_{0}, z_{0} \leq y_{0}, x_{0} \leq w_{0}$, and $z_{0} \leq w_{0}$. By the mixed monotone property of $F$, we have $x_{n} \leq y_{n}, z_{n} \leq y_{n}, x_{n} \leq w_{n}$, and $z_{n} \leq w_{n}$ for all $n \in \mathbf{N}$. Thus, by (2.1), we have

$$
\begin{aligned}
& d\left(y_{n+1}, x_{n+1}\right):=d\left(F\left(y_{n}, z_{n}, w_{n}, x_{n}\right), F\left(x_{n}, y_{n}, z_{n}, w_{n}\right)\right) \\
& \leq \phi\left(\max \left\{d\left(y_{n}, x_{n}\right), d\left(z_{n}, y_{n}\right), d\left(w_{n}, z_{n}\right), d\left(x_{n}, w_{n}\right)\right\}\right), \\
& d\left(y_{n+1}, z_{n+1}\right):=d\left(F\left(y_{n}, z_{n}, w_{n}, x_{n}\right), F\left(z_{n}, w_{n}, x_{n}, y_{n}\right)\right) \\
& \leq \phi\left(\max \left\{d\left(y_{n}, z_{n}\right), d\left(z_{n}, w_{n}\right), d\left(w_{n}, x_{n}\right), d\left(x_{n}, y_{n}\right)\right\}\right), \\
& d\left(w_{n+1}, x_{n+1}\right):=d\left(F\left(w_{n}, x_{n}, y_{n}, z_{n}\right), F\left(x_{n}, y_{n}, z_{n}, w_{n}\right)\right) \leq \phi\left(\max \left\{d\left(x_{n}, w_{n}\right), d\left(y_{n}, x_{n}\right), d\left(z_{n}, y_{n}\right), d\left(w_{n}, z_{n}\right)\right\}\right), \\
& d\left(w_{n+1}, z_{n+1}\right):=d\left(F\left(w_{n}, x_{n}, y_{n}, z_{n}\right), F\left(z_{n}, w_{n}, x_{n}, y_{n}\right)\right) \\
& \leq \phi\left(\max \left\{d\left(z_{n}, w_{n}\right), d\left(w_{n}, x_{n}\right), d\left(x_{n}, y_{n}\right), d\left(y_{n}, z_{n}\right)\right\}\right) .
\end{aligned}
$$

By (2.23) and (2.26), we have

$$
\begin{aligned}
\max & \left\{d\left(y_{n+1}, x_{n+1}\right), d\left(y_{n+1}, z_{n+1}\right), d\left(w_{n+1}, x_{n+1}\right), d\left(w_{n+1}, z_{n+1}\right)\right\} \\
& \leq \phi\left(\max \left\{d\left(y_{n}, x_{n}\right), d\left(y_{n}, z_{n}\right), d\left(w_{n}, x_{n}\right), d\left(w_{n}, z_{n}\right)\right\}\right) \\
& \leq \phi^{2}\left(\max \left\{d\left(y_{n-1}, x_{n-1}\right), d\left(y_{n-1}, z_{n-1}\right), d\left(w_{n-1}, x_{n-1}\right), d\left(w_{n-1}, z_{n-1}\right)\right\}\right) \\
& \vdots \\
& \leq \phi^{n+1}\left(\max \left\{d\left(y_{0}, x_{0}\right), d\left(y_{0}, z_{0}\right), d\left(w_{0}, x_{0}\right), d\left(w_{0}, z_{0}\right)\right\}\right) .
\end{aligned}
$$

By letting $n \rightarrow+\infty$ in (2.27) and using the property of $\phi$ and the fact that $d$ is continuous on its variable, we get that $\max \{d(y, x), d(y, z), d(w, x), d(w, z)\}=0$. Hence, $y=z=x=w$.

Corollary 2.8. In addition to the hypotheses of Corollary 2.3 (resp., Corollary 2.5), suppose that

$$
\left[\left(x_{0} \leq y_{0}\right) \wedge\left(z_{0} \leq y_{0}\right) \wedge\left(x_{0} \leq w_{0}\right) \wedge\left(z_{0} \leq w_{0}\right)\right] \vee\left[\left(y_{0} \leq x_{0}\right) \wedge\left(y_{0} \leq z_{0}\right) \wedge\left(w_{0} \leq x_{0}\right) \wedge\left(w_{0} \leq z_{0}\right)\right]
$$

Then, $x=y=z=w$.

Example 2.9. Let $X=[0,1]$ with usual order. Define $d: X \times X \rightarrow X$ by $d(x, y)=|x-y|$. Define $F: X^{4} \rightarrow X$ by

$$
F(x, y, z, w)= \begin{cases}0, & \max \{y, w\} \geq \min \{x, z\} \\ \frac{1}{4}(\min \{x, z\}-\max \{y, w\}), & \max \{y, w\}<\min \{x, z\}\end{cases}
$$


Then,

(a) $(X, d, \leq)$ is a complete ordered metric space,

(b) for $x, y, z, w, u, v, h, l \in X$ with $x \geq u, y \leq v, z \geq h$, and $w \leq l$, we have that

$$
d(F(x, y, z, w), F(u, v, h, l)) \leq \frac{1}{2} \max \{d(x, u), d(y, v), d(z, h), d(w, l)\}
$$

(c) holds for all $x \geq u, y \leq v, z \geq h$, and $w \leq l$,

(d) F has the mixed monotone property.

Proof. To prove $(b)$, given $x, y, z, w, u, v, h, l \in X$ with $x \geq u, y \leq v, z \geq h$, and $w \leq l$, we examine the following cases.

Case 1. If $\max \{y, w\} \geq \min \{x, z\}$, and $\max \{v, l\} \geq \min \{u, w\}$. Here, we have

$$
d(F(x, y, z, w), F(u, v, h, l))=0 \leq \frac{1}{2} \max \{d(x, u), d(y, v), d(z, h), d(w, l)\} .
$$

Case 2. If $\max \{y, w\} \geq \min \{x, z\}$ and $\max \{v, l\}<\min \{u, h\}$. This case is impossible since

$$
\begin{gathered}
y \leq v<\min \{u, h\} \leq \min \{x, z\} \\
w \leq l<\min \{u, h\} \leq \min \{x, z\} .
\end{gathered}
$$

So,

$$
\max \{y, w\}<\min \{x, z\}
$$

Case 3. If $\max \{y, w\}<\min \{x, z\}$ and $\max \{v, l\} \geq \min \{u, h\}$.

This case will have different possibilities. hence

(i) Let $\max \{y, w\}=y$ and $\max \{v, l\}=v$. Suppose that $h \leq v$; then $h-y \leq v-y$ and

$$
\begin{aligned}
\min \{x, z\}-\max \{y, w\} & =\min \{x, z\}-y \\
& \leq z-y=z-h+h-y \\
& \leq z-h+v-y=d(z, h)+d(y, v) \\
& \leq 2 \max \{d(x, u), d(y, v), d(z, h), d(w, l)\} .
\end{aligned}
$$


Therefore,

$$
\begin{aligned}
d(F(x, y, z, w), F(u, v, h, l)) & =d\left(\frac{1}{4}(\min \{x, z\}-\max \{y, w\}), 0\right) \\
& =\frac{1}{4}(\min \{x, z\}-y) \\
& \leq \frac{1}{2} \max \{d(x, u), d(y, v), d(z, h), d(w, l)\}
\end{aligned}
$$

Suppose that $u \leq v$; then $u-y \leq v-y$ and hence

$$
\begin{aligned}
\min \{x, z\}-\max \{y, w\} & =\min \{x, z\}-y \\
& \leq x-y=x-u+u-y \\
& \leq(x-u)+(v-y)=d(x, u)+d(v, y) \\
& \leq 2 \max \{d(x, u), d(y, v), d(z, h), d(w, l)\} .
\end{aligned}
$$

Therefore,

$$
\begin{aligned}
d(F(x, y, z, w), F(u, v, h, l)) & =d\left(\frac{1}{4}(\min \{x, z\}-\max \{y, w\}), 0\right) \\
& =\frac{1}{4}(\min \{x, z\}-y) \\
& \leq \frac{1}{2} \max \{d(x, u), d(y, v), d(z, h), d(w, l)\} .
\end{aligned}
$$

(ii)Let $\max \{y, w\}=y$ and $\max \{v, l\}=l$. Suppose that $h \leq l$; then $h-y \leq l-y$ and (since $w \leq y$ ) hence

$$
\begin{aligned}
\min \{x, z\}-\max \{y, w\} & =\min \{x, z\}-y \\
& \leq z-y=z-h+h-y \\
& \leq z-h+l-y \leq z-h+l-w=d(z, h)+d(w, l) \\
& \leq 2 \max \{d(x, u), d(y, v), d(z, h), d(w, l)\} .
\end{aligned}
$$

Therefore,

$$
\begin{aligned}
d(F(x, y, z, w), F(u, v, h, l)) & =d\left(\frac{1}{4}(\min \{x, z\}-\max \{y, w\}), 0\right) \\
& =\frac{1}{4}(\min \{x, z\}-y) \\
& \leq \frac{1}{2} \max \{d(x, u), d(y, v), d(z, h), d(w, l)\}
\end{aligned}
$$


Suppose that $u \leq l$; then $u-y \leq l-y$ and (since $w \leq y$ ) hence

$$
\begin{aligned}
\min \{x, z\}-\max \{y, w\} & \min \{x, z\}-y \\
& \leq x-y=x-u+u-y \\
& \leq(x-u)+(l-y) \leq x-u+l-w=d(x, u)+d(w, l) \\
& \leq 2 \max \{d(x, u), d(y, v), d(z, h), d(w, l)\} .
\end{aligned}
$$

Therefore,

$$
\begin{aligned}
d(F(x, y, z, w), F(u, v, h, l)) & =d\left(\frac{1}{4}(\min \{x, z\}-\max \{y, w\}), 0\right) \\
& =\frac{1}{4}(\min \{x, z\}-y) \\
& \leq \frac{1}{2} \max \{d(x, u), d(y, v), d(z, h), d(w, l)\}
\end{aligned}
$$

(iii)Let $\max \{y, w\}=w$ and $\max \{v, l\}=v$. Suppose that $h \leq v$; then $h-w \leq v-w$, but $y \leq w$, and hence

$$
\begin{aligned}
\min \{x, z\}-\max \{y, w\} & =\min \{x, z\}-w \\
& \leq z-w=z-h+h-w \\
& \leq z-h+v-w \leq z-h+v-y=d(z, h)+d(y, v) \\
& \leq 2 \max \{d(x, u), d(y, v), d(z, h), d(w, l)\} .
\end{aligned}
$$

Therefore,

$$
\begin{aligned}
d(F(x, y, z, w), F(u, v, h, l)) & =d\left(\frac{1}{4}(\min \{x, z\}-\max \{y, w\}), 0\right) \\
& =\frac{1}{4}(\min \{x, z\}-w) \\
& \leq \frac{1}{2} \max \{d(x, u), d(y, v), d(z, h), d(w, l)\}
\end{aligned}
$$

Suppose that $u \leq v$; then $u-w \leq v-w$ and hence

$$
\begin{aligned}
\min \{x, z\}-\max \{y, w\} & =\min \{x, z\}-w \\
& \leq x-w=x-u+u-w \\
& \leq(x-u)+(v-w) \leq x-u+v-y=d(x, u)+d(v, y) \\
& \leq 2 \max \{d(x, u), d(y, v), d(z, h), d(w, l)\}
\end{aligned}
$$


Therefore,

$$
\begin{aligned}
d(F(x, y, z, w), F(u, v, h, l)) & =d\left(\frac{1}{4}(\min \{x, z\}-\max \{y, w\}), 0\right) \\
& =\frac{1}{4}(\min \{x, z\}-w) \\
& \leq \frac{1}{2} \max \{d(x, u), d(y, v), d(z, h), d(w, l)\}
\end{aligned}
$$
hence

(iv)Let $\max \{y, w\}=w$ and $\max \{v, l\}=l$. Suppose that $h \leq l$; then $h-w \leq l-w$ and

$$
\begin{aligned}
\min \{x, z\}-\max \{y, w\} & =\min \{x, z\}-w \\
& \leq z-w=z-h+h-w \\
& \leq z-h+l-w=d(z, h)+d(w, l) \\
& \leq 2 \max \{d(x, u), d(y, v), d(z, h), d(w, l)\} .
\end{aligned}
$$

Therefore,

$$
\begin{aligned}
d(F(x, y, z, w), F(u, v, h, l)) & =d\left(\frac{1}{4}(\min \{x, z\}-\max \{y, w\}), 0\right) \\
& =\frac{1}{4}(\min \{x, z\}-w) \\
& \leq \frac{1}{2} \max \{d(x, u), d(y, v), d(z, h), d(w, l)\} .
\end{aligned}
$$

Suppose that $u \leq l$; then $u-w \leq l-w$ and hence

$$
\begin{aligned}
\min \{x, z\}-\max \{y, w\} & =\min \{x, z\}-w \\
& \leq x-w=x-u+u-w \\
& \leq(x-u)+(l-w)=d(x, u)+d(w, l) \\
& \leq 2 \max \{d(x, u), d(y, v), d(z, h), d(w, l)\} .
\end{aligned}
$$

Therefore,

$$
\begin{aligned}
d(F(x, y, z, w), F(u, v, h, l)) & =d\left(\frac{1}{4}(\min \{x, z\}-\max \{y, w\}), 0\right) \\
& =\frac{1}{4}(\min \{x, z\}-w) \\
& \leq \frac{1}{2} \max \{d(x, u), d(y, v), d(z, h), d(w, l)\}
\end{aligned}
$$


Case 4. (i)If $\max \{y, w\}<\min \{x, z\}$ and $\max \{v, l\}<\min \{u, h\}$.

Since $x \geq u$ and $z \geq h$, then $\min \{x, z\} \geq \min \{u, h\}$, and also since $y \geq v$ and $w \geq l$, then $\max \{v, l\} \geq \max \{y, w\}$. Thus,

$$
\begin{aligned}
d(F(x, y, z), F(u, v, w)) & =d\left(\frac{1}{4}(\min \{x, z\}-\max \{y, w\}), \frac{1}{4}(\min \{u, h\}-\max \{v, l\})\right) \\
& =\frac{1}{4}|(\min \{x, z\}-\min \{u, h\})+(\max \{v, l\}-\max \{y, w\})| .
\end{aligned}
$$

(ii)If $\min \{u, h\}=u$ and $\max \{v, l\}=v$, then $\min \{x, z\}-\min \{u, h\} \leq x-u$ and $\max \{v, l\}-\max \{y, w\} \leq v-y$. Thus,

$$
\begin{aligned}
d(F(x, y, z, w), F(u, v, h, l)) & \leq \frac{1}{4}[(x-u)+(v-y)] \\
& =\frac{1}{4}[d(x, u)+d(y, v)] \\
& \leq \frac{1}{2} \max \{d(x, u), d(y, v), d(z, h), d(w, l)\} .
\end{aligned}
$$

(iii)If $\min \{u, h\}=h$ and $\max \{v, l\}=v$, then $\min \{x, z\}-\min \{u, h\} \leq z-h$ and $\max \{v, l\}-\max \{y, w\} \leq v-y$, hence

$$
\begin{aligned}
d(F(x, y, z, w), F(u, v, h, l)) & \leq \frac{1}{4}[(z-h)+(v-y)] \\
& =\frac{1}{4}[d(z, h)+d(y, v)] \\
& \leq \frac{1}{2} \max \{d(x, u), d(y, v), d(z, h), d(w, l)\} .
\end{aligned}
$$

(iv)If $\min \{u, h\}=u$ and $\max \{v, l\}=l$, then $\min \{x, z\}-\min \{u, h\} \leq x-u$ and $\max \{v, l\}-\max \{y, w\} \leq l-w$, and hence

$$
\begin{aligned}
d(F(x, y, z, w), F(u, v, h, l)) & \leq \frac{1}{4}[(x-u)+(l-w)] \\
& =\frac{1}{4}[d(x, u)+d(w, l)] \\
& \leq \frac{1}{2} \max \{d(x, u), d(y, v), d(z, h), d(w, l)\} .
\end{aligned}
$$


(v)If $\min \{u, h\}=h$ and $\max \{v, l\}=l$, then $\min \{x, z\}-\min \{u, h\} \leq z-h$ and $\max \{v, l\}-$ $\max \{y, w\} \leq l-w$, and hence

$$
\begin{aligned}
d(F(x, y, z, w), F(u, v, h, l)) & \leq \frac{1}{4}[(z-h)+(l-w)] \\
& =\frac{1}{4}[d(z, h)+d(w, l)] \\
& \leq \frac{1}{2} \max \{d(x, u), d(y, v), d(z, h), d(w, l)\} .
\end{aligned}
$$

To prove (c), let $x, y, z, w \in X$. To show that $F(x, y, z, w)$ is monotone nondecreasing in $x$, let $x_{1}, x_{2} \in X$ with $x_{1} \leq x_{2}$.

If $\max \{y, w\} \geq \min \left\{x_{1}, z\right\}$, then $F\left(x_{1}, y, z, w\right)=0 \leq F\left(x_{2}, y, z, w\right)$. If $\max \{y, w\}<$ $\min \left\{x_{1}, z\right\}$, then

$$
F\left(x_{1}, y, z, w\right)=\frac{1}{4}\left(\min \left\{x_{1}, z\right\}-\max \{y, w\}\right) \leq \frac{1}{4}\left(\min \left\{x_{2}, z\right\}-\max \{y, w\}\right)=F\left(x_{2}, y, z, w\right) .
$$

Therefore, $F(x, y, z, w)$ is monotone nondecreasing in $x$. Similarly, we may show that $F(x, y, z, w)$ is monotone nondecreasing in $z$.

To show that $F(x, \mathrm{y}, z, w)$ is monotone nonincreasing in $y$, let $y_{1}, y_{2} \in X$ with $y_{1} \leq y_{2}$. If $\max \left\{y_{2}, w\right\} \geq \min \{x, z\}$, then $F\left(x, y_{2}, z, w\right)=0 \leq F\left(x_{1}, y, z, w\right)$. If $\max \left\{y_{2}, w\right\}<\min \{x, z\}$, then

$$
F\left(x, y_{2}, z, w\right)=\frac{1}{4}\left(\min \{x, z\}-\max \left\{y_{2}, w\right\}\right) \leq \frac{1}{4}\left(\min \{x, z\}-\max \left\{y_{1}, w\right\}\right)=F\left(x, y_{2}, z, w\right)
$$

Therefore, $F(x, y, z, w)$ is monotone nonincreasing in $y$. Similarly, we may show that $F(x, y, z, w)$ is monotone nonincreasing in $w$.

Thus, by Theorem 2.1 (let $\phi(t)=(t / 2)), F$ has a unique quadruple fixed point, namely, $(0,0,0,0)$. Since the condition of Theorem 2.7 is satisfied, $(0,0,0,0)$ is the unique quadruple fixed point of $F$.

Remark 2.10. We notice that for, $F: X^{2 n} \rightarrow X, \quad(n \in \mathbb{N})$, it is very natural to consider the analog of Theorem 2.1-Theorem 2.7 to get fixed points. Moreover, for $F: X^{2 n+1} \rightarrow X \quad(n \in$ $\mathbb{N})$, the analog of Theorem 7-Theorem 11 of Berinde and Borcut [2] yields fixed points.

\section{References}

[1] T. Gnana Bhaskar and V. Lakshmikantham, "Fixed point theorems in partially ordered metric spaces and applications," Nonlinear Analysis, vol. 65, no. 7, pp. 1379-1393, 2006.

[2] V. Berinde and M. Borcut, "Tripled fixed point theorems for contractive type mappings in partially ordered metric spaces," Nonlinear Analysis, vol. 74, no. 15, pp. 4889-4897, 2011.

[3] V. Lakshmikantham and L. Ćirić, "Coupled fixed point theorems for nonlinear contractions in partially ordered metric spaces," Nonlinear Analysis, vol. 70, no. 12, pp. 4341-4349, 2009. 
[4] M. Abbas, H. Aydi, and E. Karapınar, "Tripled fixed points of multivalued nonlinear contraction mappings in partially ordered metric spaces," Abstract and Applied Analysis, vol. 2011, Article ID 812690, 12 pages, 2011.

[5] M. Abbas, M. Ali Khan, and S. Radenović, "Common coupled fixed point theorems in cone metric spaces for $\phi$-compatible mappings," Applied Mathematics and Computation, vol. 217, no. 1, pp. 195-202, 2010.

[6] M. Abbas, A. R. Khan, and T. Nazir, "Coupled common fixed point results in two generalized metric spaces," Applied Mathematics and Computation, vol. 217, no. 13, pp. 6328-6336, 2011.

[7] H. Aydi, B. Damjanović, B. Samet, and W. Shatanawi, "Coupled fixed point theorems for nonlinear contractions in partially ordered G-metric spaces," Mathematical and Computer Modelling, vol. 54, no. 9-10, pp. 2443-2450, 2011.

[8] H. Aydi, E. Karapınar, and W. Shatanawi, "Coupled fixed point results for $(\psi, \varphi)$-weakly contractive condition in ordered partial metric spaces," Computers \& Mathematics with Applications, vol. 62, no. 12, pp. 4449-4460, 2011.

[9] B. S. Choudhury and P. Maity, "Coupled fixed point results in generalized metric spaces," Mathematical and Computer Modelling, vol. 54, no. 1-2, pp. 73-79, 2011.

[10] E. Karapınar, "Couple fixed point theorems for nonlinear contractions in cone metric spaces," Computers E Mathematics with Applications, vol. 59, no. 12, pp. 3656-3668, 2010.

[11] N. V. Luong and N. X. Thuan, "Coupled fixed points in partially ordered metric spaces and application," Nonlinear Analysis, vol. 74, no. 3, pp. 983-992, 2011.

[12] H. K. Nashine and W. Shatanawi, "Coupled common fixed point theorems for a pair of commuting mappings in partially ordered complete metric spaces," Computers E Mathematics with Applications, vol. 62, no. 4, pp. 1984-1993, 2011.

[13] F. Sabetghadam, H. P. Masiha, and A. H. Sanatpour, "Some coupled fixed point theorems in cone metric spaces," Fixed Point Theory and Applications, vol. 2009, Article ID 125426, 8 pages, 2009.

[14] B. Samet, "Coupled fixed point theorems for a generalized Meir-Keeler contraction in partially ordered metric spaces," Nonlinear Analysis, vol. 72, no. 12, pp. 4508-4517, 2010.

[15] B. Samet and C. Vetro, "Coupled fixed point, F-invariant set and fixed point of N-order," Annals of Functional Analysis, vol. 1, no. 2, pp. 46-56, 2010.

[16] B. Samet and H. Yazidi, "Coupled fixed point theorems in partially ordered $\varepsilon$-chainable metric spaces," TJMCS, vol. 1, no. 30, pp. 142-151, 2010.

[17] S. Sedghi, I. Altun, and N. Shobe, "Coupled fixed point theorems for contractions in fuzzy metric spaces," Nonlinear Analysis, vol. 72, no. 3-4, pp. 1298-1304, 2010.

[18] W. Shatanawi, B. Samet, and M. Abbas, "Coupled fixed point theorems for mixed monotone mappings in ordered partial metric spaces," Mathematical and Computer Modelling, vol. 55, no. 3-4, pp. 680-687, 2012.

[19] W. Shatanawi, "Some common coupled fixed point results in cone metric spaces," International Journal of Mathematical Analysis, vol. 4, no. 45-48, pp. 2381-2388, 2010.

[20] W. Shatanawi, "Partially ordered cone metric spaces and coupled fixed point results," Computers $\mathcal{E}$ Mathematics with Applications, vol. 60, no. 8, pp. 2508-2515, 2010.

[21] W. Shatanawi, "Fixed point theorems for nonlinear weakly $C$-contractive mappings in metric spaces," Mathematical and Computer Modelling, vol. 54, no. 11-12, pp. 2816-2826, 2011.

[22] Z. Golubović, Z. Kadelburg, and S. Radenović, “Coupled coincidence points of mappings in ordered partial metric spaces," Abstract and Applied Analysis, vol. 2012, Article ID 192581, 18 pages, 2012.

[23] D. Dorić, Z. Kadelburg, and S. Radenović, "Coupled fixed point results for mappings without mixed monotone property," Applied Mathematics Letters. In press.

[24] H. K. Nashine, Z. Kadelburg, and S. Radenović, "Coupled common fixed point theorems for $w^{*}$ compatible mappings in ordered cone metric spaces," Applied Mathematics and Computation, vol. 218, no. 9, pp. 5422-5432, 2012.

[25] Z. Kadelburg and S. Radenović, "Coupled fixed point results under TVS-cone metric and W-conedistance," Advence Fixed Point Theory, vol. 2, no. 1, pp. 29-46, 2012.

[26] Y. J. Cho, Z. Kadelburg, R. Saadati, and W. Shatanawi, "Coupled fixed point theorems under weak contractions," Discrete Dynamics in Nature and Society, vol. 2012, Article ID 184534, 9 pages, 2012.

[27] W. Long, B. E. Rhoades, and M. Rajović, "Coupled coincidence points for two mappings in metric spaces and cone metric spaces," Fixed Point Theory and Applications, vol. 2012, article 12, 2012.

[28] Z. M. Fadail and A. G. B. Ahmad, "Coupled Fixed point theorems of single-valued mappings for c-distanse in cone metric spaces," Journal of Applied Mathematics. In press. 
[29] Z. M. Fadail, A. G. B. Ahmad, and Z. Golubović, "Fixed point theorems of single-valued mapping for $c$-distance in cone metric space," Abstract and Applied Analysis. In press.

[30] W. Sintunavarat, Y. Je, and P. Kumam, "Coupled Fixed point theorem for weak contraction mapping under F-invariant set," Abstract and Applied Analysis, vol. 2012, Article ID 324874, 15 pages, 2012.

[31] A. Razani, H. H. Zadeh, and A. Jabbari, "Coupled fixed point theorems in partially ordered metric spaces which endowed with vector-valued metrics," Australian Journal of Basic and Applied Sciences, vol. 6, no. 2, pp. 124-129, 2012.

[32] M. Abbas, "Wutiphol sintunavarat, and poom kumam, coupled fixed of generalized contractive mapping on partially ordered G-metric spaces," Fixed Point Theory and Applications, vol. 2012, article 31, 2012.

[33] E. Karapınar, “Quartet fixed point for nonlinear contraction,” http://arxiv.org/abs/1106.5472.

[34] E. Karapınar and N. V. Luong, "Quadruple fixed point theorems for nonlinear contractions," Computers and Mathematics with Applications. In press.

[35] E. Karapinar and V. Berinde, "Quadruple fixed point theorems for nonlinear contractions in partially ordered metric spaces," Banach Journal of Mathematical Analysis, vol. 6, no. 1, pp. 74-89, 2012.

[36] E. Karapınar, "Quadruple fixed point theorems for weak -contractions," ISRN Mathematical Analysis, vol. 2011, Article ID 989423, 15 pages, 2011.

[37] E. Karapınar, H. Aydi, and I. S. Yüce, "Quadruple fixed point theorems in partially ordered metric spaces depending on another function," ISRN Mathematical Analysis. In press.

[38] E. Karapinar, "A new quartet fixed point theorem for nonlinear contractions," JP Journal of Fixed Point Theory and Applications, vol. 6, no. 2, pp. 119-135, 2011.

[39] Z. Mustafa, H. Aydi, and E. Karapınar, "Mixed g-monotone property and quadruple fixed point theorems in partially ordered metric spaces," Fixed Point Theory and Applications, vol. 2012, article 71, 2012.

[40] J. Matkowski, "Fixed point theorems for mappings with a contractive iterate at a point," Proceedings of the American Mathematical Society, vol. 62, no. 2, pp. 344-348, 1977. 


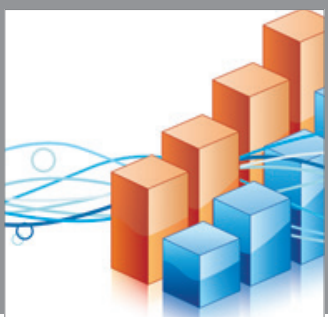

Advances in

Operations Research

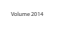

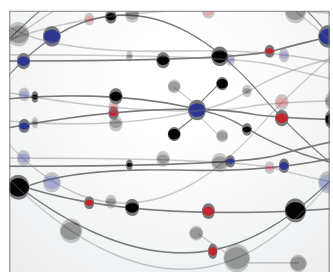

\section{The Scientific} World Journal
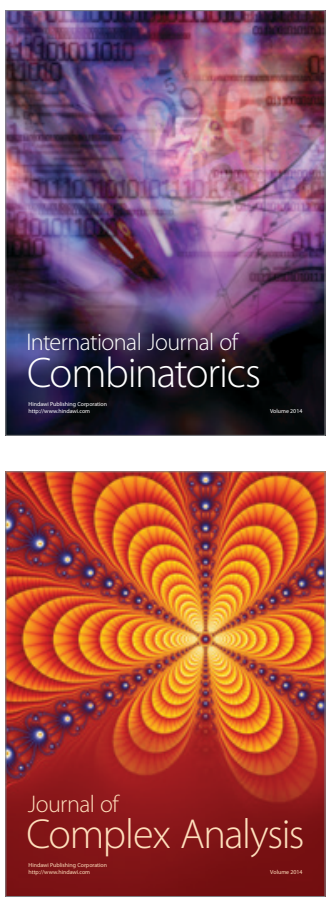

International Journal of

Mathematics and

Mathematical

Sciences
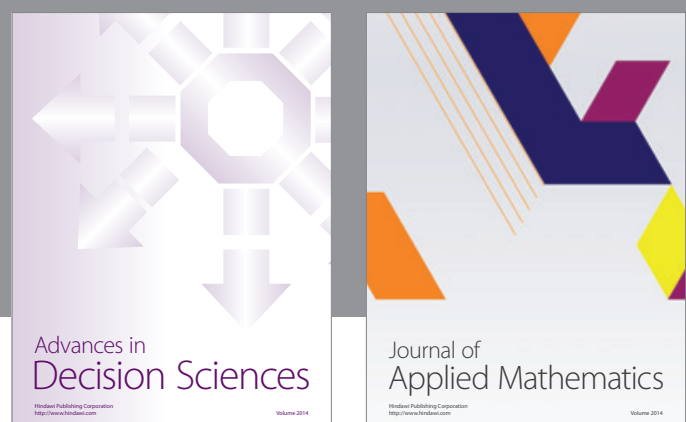

Journal of

Applied Mathematics
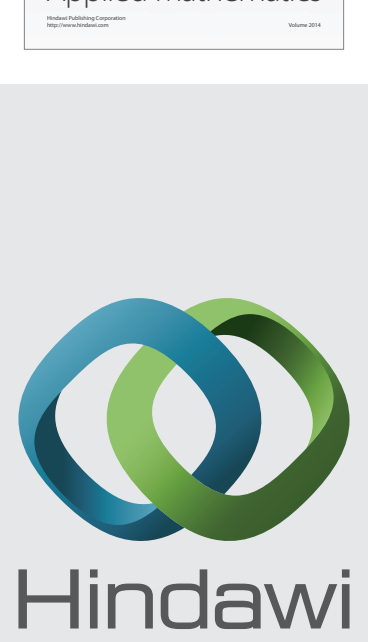

Submit your manuscripts at http://www.hindawi.com
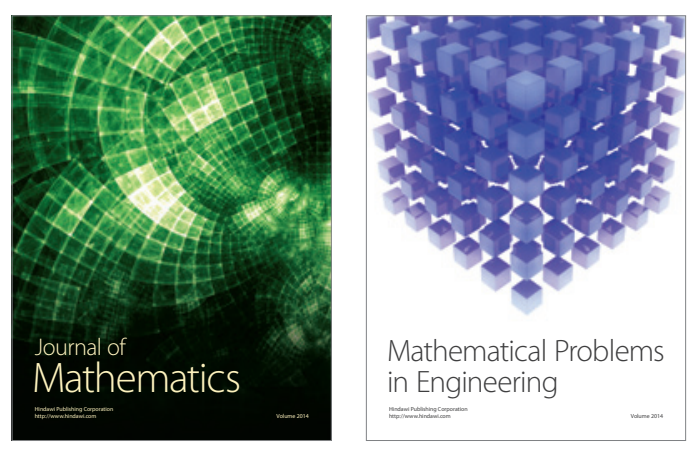

Mathematical Problems in Engineering
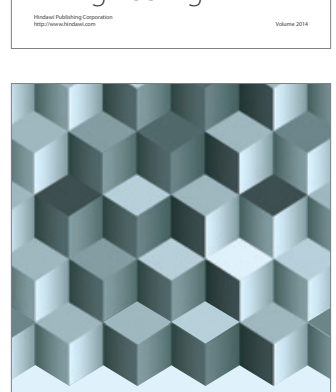

Journal of

Function Spaces
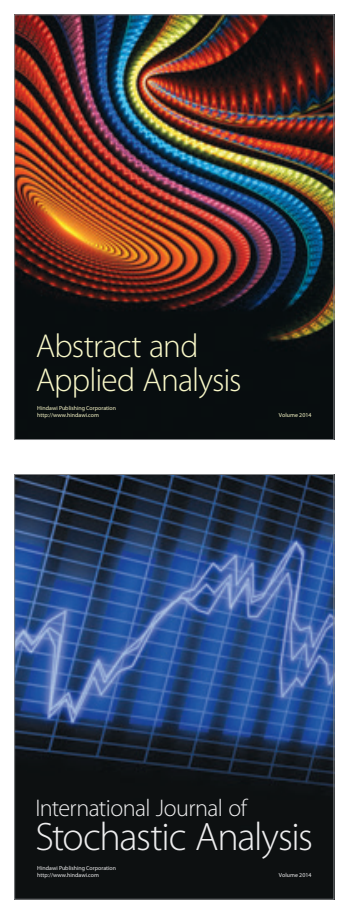

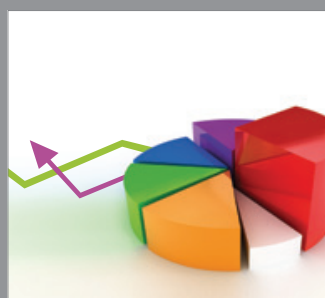

ournal of

Probability and Statistics

Promensencen
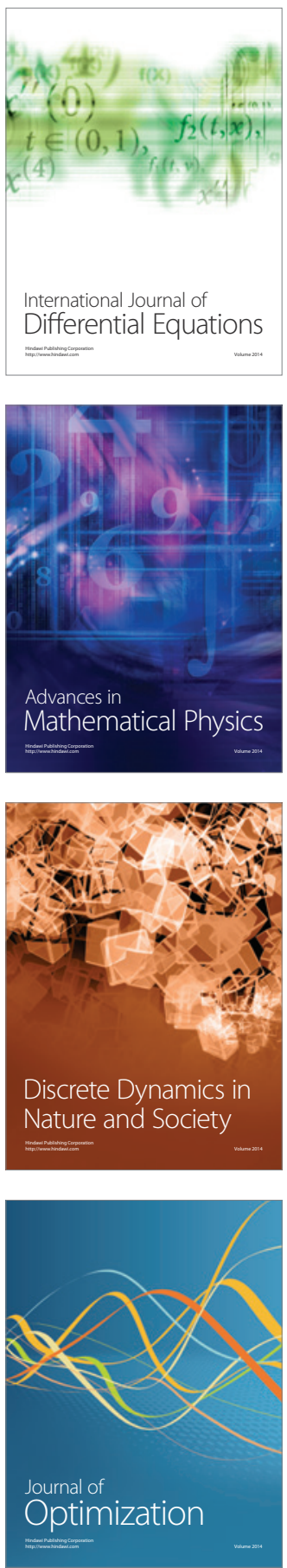